\title{
Algorithmic processing to aid in leukemia detection
}

\section{Type of article: conference Abstract}

\author{
Abdellatif BOUZID-DAHO ${ }^{1}$, Mohamed BOUGHAZI ${ }^{2}$, Camel TANOUGAST $^{3}$ \\ 1, 2 Department of electronic, Faculty of Engineering Sciences, Laboratory of study and \\ research in instrumentation and communication of Annaba, University of Badji Mokhtar, \\ Annaba, Algeria \\ 3. Laboratory of conception, optimization and modeling of the systems, University of \\ Lorraine, Metz, France \\ daholion@live.fr
}

\begin{abstract}
Background: I present our medical context with some basic concepts in order to understand the results of our work, and then I begin the explanation of mathematical morphology. I will conclude by the description of algorithmic processing propose in this paper. Cancers, including leukemia and lymphoma, can cause uncontrolled growth of an abnormal type of blood cell in the bone marrow, resulting in a greatly increased risk for infection and or serious bleeding.

Methods: We present detailed steps of our proposed systems, to obtain a final result that shows the detection of abnormal cells. It typically starts with a median filter pre-processing step and then applies different morphologic operator, which allows us to segment the original image and detect cancerous cells. The basic idea behind all the operators in the mathematical morphology is to compare the set of objects to analyze another object of known form, which is called a structuring element. The structuring element is a geometric figure, simple to form, known or arbitrary, and can be a circle, segment, square, or triangle.

Results: We show the different results obtained after testing carried out in algorithmic processing using MATLAB: To ameliorate the visualization of the abnormal blood cells, we have applied the elements basis morphological operations in a different way. We have performed an opening by reconstruction and a closing by reconstruction. The obtained result show that we have obtained an efficient detection of the targeted objects (abnormal blood cells or leukemia).

Conclusion: In this paper, we have utilized the operators of the mathematical morphology with the aim to detect abnormal cells for diagnostic aid and transmission of accurate and precise clinical information, which helps specialists in medicine (hematologists) to distinguish abnormal cells or cancerous and to follow the evolution of leukemia. The algorithmic processing presented in this article has been able to perform the task of detection of cancerous cells with success; it has produced remarkable and satisfactory results. We think of the future concept as a system of aid for diagnosis from microelectronics integration to the base of reconfigurable technologies applied to cells for the goal of quantification of the cancer region.
\end{abstract}

Keywords: Diagnostic, Algorithmic, Blood Cells, Leukemia, Morphological Operator.

\section{Declaration of conflicts}

This article was selected from ICHSMT'16 abstract book. 


\section{Authors' biography}

No Biography

3. References

No reference 